Pacific Journal of Mathematics

LUMPY SUBSETS IN LEFT-AMENABLE LOCALLY COMPACT
SEMIGROUPS

SEM 


\title{
LUMPY SUBSETS IN LEFT-AMENABLE LOCALLY COMPACT SEMIGROUPS
}

\author{
MAHLON M. DAY
}

\begin{abstract}
This note adapts to locally compact, topologically left-amenable semigroups $S$ the characterization given by Mitchell in discrete semigroups of those subsets $T$ of $S$ substantial enough that at least one topologically left-invariant mean in $S$ is concentrated in $T$.
\end{abstract}

Introduction. Mitchell (1965) showed that for a discrete leftamenable semigroup $S$ the following two properties of a subset $T$ of $S$ are equivalent:

(1) There is at least one left-invariant mean $\alpha$ on the space $m(S)$ of bounded functions on $S$ which is concentrated on $T$; that is, $\alpha\left(\xi_{T}\right)=1$.

(2) $T$ is left-thick; that is, for each finite subset $\sigma$ of $S$ there is an $S$ in $S$ such that $\sigma s=\{t s \mid t \in \sigma\}$ is contained in $T$.

My paper (Day, 1969, Th. 7.2) gave a version of this for locally compact groups, which Wong (preceding paper) points out has a misprint; $K \backslash E s$ should have been $K \cap E s$ in condition (2) of that theorem.

In a locally compact group $G$ there are (at least) three conditions equivalent to left amenability of $G$; that is, existence of a left-invariant mean $\alpha$ on the bounded measurable functions on $G$. The pertinent three are called in Day (1968) conditions (lsau), (lsam), and (lsa $\pi$ ) and will be defined in the next section. In locally compact semigroups each implies the next, but they are not known to be equivalent except in groups. In the preceding paper Wong chose a condition $(W)$ equivalent to (lsau) and generalized Mitchell's theorem to semigroups satisfying $(W)$. This note picks the simpler middle property (lsam) and proves a Mitchelloid theorem for such semigroups. For the weakest of the properties (lsa $\pi$ ) some implications are given but no equivalent condition is yet known.

Notation. $C_{0}$ is the space of continuous functions vanishing at infinity on $S$, a locally compact semigroup, and $M$ is the space of regular Borel measures on $S$. [It is well known that $M$ is like the conjugate space of $C_{0}$; see Hewitt and Ross (1963).] $P \subset M$ is the set of probability measures and $P_{c} \subset P$ is the set of probability measures with compact support, so $P_{c}$ is dense in $P . \quad P^{* *} \subset M^{* *}$ is the set of means on $M^{*}$; as usual, the canonical image $Q\left(P_{c}\right)$ of $P_{c}$ into $M^{* *}$ is $w^{*}$-dense in $P^{* *}$. $C_{0}$ and $M^{*}$ are abstract $M$-spaces and $M$ and $M^{* *}$ are abstract 
$L$-spaces. $\quad M$ is a Banach algebra under the usual convolution operation: For each $x$ in $C_{0}$,

$$
[\nu * \mu](x)=[\nu \times \mu]\left(x^{\vee}\right)
$$

where $x^{\vee}$ is defined on $S \times S$ by: For each $(s, t)$ in $S \times S$,

$$
x^{\vee}(s, t)=x(s t) \text {. }
$$

Arens multiplication can then be defined (see, for example, Day (1969)) to make $M^{* *}$ also a Banach algebra for which the natural isometric embedding $Q$ of $M$ into $M^{* *}$ becomes an algebra isomorphism.

If $T$ is a Borel subset of $S$, the characteristic functional $\chi_{T}$ of $T$ is that element of $M^{*}$ defined by: $\chi_{T}(\mu)=\mu(T)$ for each $\mu$ in $M$. To state that a $\Gamma$ in $P^{* *}$ is concentrated on $T$ is to say that $\Gamma\left(\chi_{T}\right)=1$.

A locally compact semigroup $S$ will be called left-amenable if

(la $\pi)$ there exists a $\Gamma$ in $P^{* *}$ such that $Q_{\delta s} * \Gamma=\Gamma$ for each $s$ in $S$.

$S$ will be called topologically left-amenable if

(lam) there exists $\Gamma$ in $P^{* *}$ such that $Q \mu * \Gamma=\Gamma$ for each $\mu$ in $P_{c}$, or equivalently for each $\mu$ in $P$, or equivalently $\Delta * \Gamma=\Gamma$ for each $\Delta$ in $P^{* *}$

For locally compact groups these and the three conditions below are equivalent (Day 1968, 1969), but for locally compact semigroups we know only the obvious (lam) implies (la $\pi$ ).

Among the "strong" left-amenability conditions described for locally compact groups in Day $(1968,1969)$ are three pertinent to this paper. We adapt them for locally compact semigroups:

(lsau) (Uniform (on compact sets) left strong amenability) There exists a net $\left(\mu_{n}\right)$ of elements of $P_{c}$ such that for each compact set $K$ in $S,\left\|\delta_{s} * \mu_{n}-\mu_{n}\right\|$ tends to zero uniformly for $s$ in $K$.

(lsam) (topological left strong amenability) There exists a net $\left(\mu_{n}\right)$ in $P_{c}$ such that $\left\|\nu * \mu_{n}-\mu_{n}\right\|$ tends to zero for each $\nu$ in $P_{c}$.

(lsa $\pi$ ) (pointwise left strong amenability) There exists a net $\left(\mu_{n}\right)$ in $P_{c}$ such that $\left\|\delta_{s} * \mu_{n}-\mu_{n}\right\|$ tends to zero for each $s$ in $S$.

Clearly (lsau) is equivalent to the condition of the preceding paper: 
(W) There exists a net $\left(\mu_{n}\right)$ in $P_{c}$ such that for each compact set $K$ contained in $S,\left\|\nu * \mu_{n}-\mu_{n}\right\|$ tends to zero uniformly over all $\nu$ in $P_{c}$ which are supported in $K$.

Standard methods from earlier papers show that (la $\pi$ ) is equivalent to (lsa $\pi$ ) and that (lam) is equivalent to (lsam). Also (W) implies (lsam) implies (lsa $\pi$ ). In locally compact semigroups no more is known so Mitchell's theorem has several possible extensions. Wong's theorem works for semigroups with the property (W); for such semigroups he defines a version of left-thickness and proves it equivalent to the property of concentrating some topologically left-invariant mean. This paper assumes only that the semigroup is topologically left amenable and uses a different internal property of subsets of $S$. As a guide observe that Mitchell's property (2) of left-thickness is equivalent in discrete semigroups to: For each $\nu$ in $P$ with compact (= finite) support there is $s$ in $S$ with $\left[\nu * \delta_{s}\right](T)=1$. We choose an approximation of this for the first of the definitions below; the second is a direct analogue of Mitchell's and is much too strong. Even in Mitchell's case the word "lumpy" is more descriptive of the circumstances than is "thick".

Definition. A Borel set $T$ contained in $S$ is called topologically left-lumpy if for each $\nu$ in $P_{c}$ and each $\epsilon>0$ there is $s$ in $S$ such that $\left[\nu * \delta_{s}\right](T)>1-\epsilon . T$ is left-lumpy if for each compact set $K$ in $S$ there is $S$ in $S$ such that $K s \subset T$.

THeorem (Mitchelloid, Mark III). For each Borel set $T$ of a topologically left-amenable, locally compact semigroup $S$ the following conditions on $T$ are equivalent:

(3) There is a topologically left-invariant mean $\Gamma$ in $P^{* *}$ such that $\Gamma$ is concentrated on $T$; that is, $\Gamma\left(\chi_{T}\right)=1$.

(4) $T$ is topologically left-lumpy.

(5) For each $\nu$ in $P_{c}$ and each $\epsilon>0$ there is a $\mu$ in $P_{c}$ such that $[\nu * \mu](T)>1-\epsilon$.

Proof. (5) implies (4). If $\nu \in P_{c}$ and if $\left[\nu * \delta_{s}\right](T) \leqq 1-\epsilon$ for all $s$ in $S$, then for each $\mu$ in $P_{c},[\nu * \mu](T)=\mu(x)$, where $x(s)=\left[\nu * \delta_{s}\right](T)$ for all $s$ in $S$. But then $[\nu * \mu](T) \leqq 1-\epsilon$ for all $\mu$ in $P_{c}$. This is contrapositive of (5) implies (4).

(4) implies (3). If $\sigma=\left(\nu_{1}, \cdots, \nu_{k}\right)$ is a finite subset of $P_{c}$ and if $\epsilon>0$, by condition (4) there is a $s=s_{\epsilon, \sigma}$ in $S$ such that

$$
\left[\left(\nu_{1}+\cdots+\nu_{k}\right) / k * \delta_{s}\right](T)>1-\epsilon / k
$$

so for all $i \leqq k,\left[\nu_{i} * \delta_{s}\right](T)>1-\epsilon$. 
If $(\epsilon, \sigma)>\left(\epsilon^{\prime}, \sigma^{\prime}\right)$ means that $\epsilon<\epsilon^{\prime}$ and $\sigma \supseteq \sigma^{\prime}$, then $w^{*}$ compactness of $P^{* *}$ implies that the net $\left(Q \delta_{s_{\varepsilon, \sigma}}\right)$ has a $w^{*}$-convergent subnet $\left(Q \delta_{s_{m}}\right)$ with limit $\Gamma$ in $P^{* *}$. Then for each $\nu$ in $P_{c}$

$$
\begin{aligned}
1 & \geqq[Q \nu * \Gamma]\left(\chi_{T}\right)=[Q \nu]\left(\Gamma * \chi_{T}\right)=\left[\Gamma * \chi_{T}\right](\nu)=\Gamma\left(\chi_{T} * \nu\right) \\
& =\lim _{m}\left[Q \delta_{s_{m}}\right]\left(\chi_{T} * \nu\right)=\lim _{m}\left[\chi_{T} * \nu\right]\left(\delta_{s_{m}}\right) \\
& =\lim _{m} \chi_{T}\left(\nu * \delta_{s_{m}}\right)=\lim _{m}\left[\nu * \delta_{s_{m}}\right](T) .
\end{aligned}
$$

But

$$
\left[\nu * \delta_{s_{\epsilon, \sigma}}\right](T)>1-\epsilon \text { if } \nu \text { is in } \sigma
$$

so that the limit is 1 and $[Q \nu * \Gamma]\left(\chi_{T}\right)=1$ for all $\nu$ in $P_{c}$. By $w^{*}$-density of $Q P_{c}$ in $P^{* *}$ and the $w^{*}$-continuity of $*$ in its first variable, $[\Delta * \Gamma]\left(\chi_{T}\right)=$ 1 for all $\Delta$ in $P^{* *}$. In particular, $\Delta$ can be chosen to be a topologically left-invariant mean. Then $\Phi=\Delta * \Gamma$ is a topologically left-invariant mean such that $\Phi\left(\chi_{T}\right)=1$; that is, (3) holds if (4) does.

(3) implies (5). If $\Gamma$ is a topologically left-invariant mean such that $\Gamma\left(\chi_{T}\right)=1$, let $\left(\mu_{n}\right)$ be a net of elements of $P_{c}$ such that $w^{*}-\lim _{n} Q \mu_{n}=\Gamma$. Then for each $\nu$ in $P_{c}$, because an Arens product is $w^{*}-w^{*}$-continuous in its second variable when the first variable is a $Q \nu$,

$$
w^{*}-\lim _{n}\left(Q \nu * Q \mu_{n}-Q \mu_{n}\right)=Q \nu * \Gamma-\Gamma=0 \text {. }
$$

But $Q \mu_{n}\left(\chi_{T}\right)$ tends to $\Gamma\left(\chi_{T}\right)=1$, so

$$
\left[Q_{\nu} * Q \mu_{n}\right]\left(\chi_{T}\right)=\left[Q\left(\nu * \mu_{n}\right)\right]\left(\chi_{T}\right)=\chi_{T}\left(\nu * \mu_{n}\right)=\left[\nu * \mu_{n}\right](T),
$$

so $\left[\nu * \mu_{n}\right](T)$ tends to 1 for each $\nu$ in $P_{c}$. Hence for each $\nu$ and $\epsilon$ some $\mu_{n}$ satisfies (5).

REMARKs. (A) Although for locally compact groups it is well known that left-amenability implies strong left-amenability uniformly on compact sets and hence implies topological left-amenability (Day 1969, Th. 13.3), the space used there is not the full space $M$ of regular Borel measures but the smaller spaces $M_{a}$ of measures absolutely continuous with respect to Haar measure on $G$. Wong (preceding paper, Th. 5.2) points out that for a locally compact group $G$ there is a topologically left-invariant $\Gamma$ in $P^{* *}$ if and only if there is one in $P^{* *} \cap M_{a}^{* *}$.

(B) Wong also shows in Th. 5.2 that for a locally compact group $G$ the Reiter condition

$\left(\operatorname{lsau}_{1}\right)$ There is a net $\left(\varphi_{n}\right)$ in $P_{c} \cap M_{a}$ such that $\left\|\delta_{s} * \varphi_{n}-\varphi_{n}\right\|$ tends to zero uniformly in each compact subset $K$ of $S$, 
is equivalent when $S=G$ to his condition (W). We have already remarked that $(\mathrm{W})$ is equivalent to the condition (lsau) above in every locally compact semigroup.

(C) In those locally compact semigroups where (W) holds, Wong's Theorem 4.1 and my theorem above both characterize sets $T$ for which some topologically left-invariant mean is concentrated on $T$; hence in such semigroups his topologically left-thick sets are the same as my topologically left-lumpy sets.

(D) In a left-amenable locally compact semigroup no adequate characterization has been found for those sets on which left-invariant means can be concentrated; however something can be done like parts of the earlier theorem.

THEOREM (Mitchelloid, Mark IIIa). In a left amenable locally compact semigroup $S$ each of the following conditions on a Borel set $T$ implies the next in line.

(6) $T$ is left lumpy.

(5) (as in first theorem).

(4) $T$ is topologically left lumpy.

(7) There is a left-invariant mean $\Phi$ in $P^{* *}$ such that $\Phi\left(\chi_{T}\right)=1$.

(8) There is a net $\left(\mu_{n}\right)$ in $P_{c}$ such that $\lim _{n}\left\|\varphi * \mu_{n}-\mu_{n}\right\|=0$ for each finitely supported mean $\varphi$ on $S_{0}$ and $\lim _{n}\left[\varphi * \mu_{n}\right](T)=1$.

(2) (Mitchell's left thick) For each finite set $\sigma \subset S$ there is $s$ in $S$ such that $\sigma s \subset T$.

Proof. Left lumpy is stronger than (W), since it assures that $\left[\nu * \delta_{s}\right](T)=1$ if $\nu$ is supported in $K$. (W) implies (5) implies (4). The proof that (4) implies (3) need only be modified to choose $\Delta$ as a left-invariant mean to give a proof of (7). The proof that (7) implies (8) is like that of (3) implies (5), using finitely supported means instead of compactly supported ones on the left. For (8) implies (2) let $\sigma$ be a finite subset of $S$ and let $k$ be the number of elements of $\sigma$. Set $\varphi=\left(\sum_{s \in \sigma} \delta_{s}\right) / k$. If $\left(\mu_{n}\right)$ satisfies (7), then $\left[\varphi * \mu_{n}\right](T)$ tends to 1. As in the proof of (5) implies (4) there must be an $s_{n}$ in the support of $\mu_{n}$ such that $\left[\varphi * \delta_{s_{n}}\right](T)$ tends to 1 . But as soon as $\left[\varphi * \delta_{s_{n}}\right](T)>1-1 / k,\left[\varphi * \delta_{s_{n}}\right](T)=1$, so $\sigma s_{n} \subset T$.

We note that (7) and (8) are equivalent, but the proof that (2) implies (7) would require something like left-amenability of $S$ regarded as a discrete semigroup, which is not a common property of left-amenable locally compact semigroups.

Open problems. (A) Except in groups it is not known whether in a locally compact semigroup left-amenability implies topological left- 
amenability or whether topological left-amenability implies (W) which is equivalent to (lsau).

(B) A characterization is still needed for those subsets of a locally compact left-amenable semigroup on which a left-invariant mean can be concentrated; left-lumpy is much too strong and Mitchell's left-thick is much too weak. It should be noted, however, that left ideals in $S$ are left-lumpy and therefore support left-invariant means.

\section{REFERENCES}

M. M. Day, Amenability and equicontinuity, Studia Math., 31 (1968), 481-494.

Semigroups and amenability. Pages 5 to 54 in Semigroups, Proceedings of a Symposium, edited by K. W. Folley. Academic Press, New York and London, (1969).

—- Normed Linear Spaces, 3rd Edition. Springer-Verlag, Berlin-Heidelberg-New York, (1973).

T. Mitchell, Constant functions and left-invariant means on semigroups, Trans. Amer. Math. Soc., 119 (1965), 244-261.

J. C. S. Wong, A characterization of topological left-thick subsets in locally compact left-amenable semigroups. (1976). This Journal, immediately preceding this note.

Received December 12, 1975.

UNIVERSITY OF ILLINOIS - URBANA 



\section{Pacific Journal of Mathematics}

\section{Vol. 62, No. $1 \quad$ January, 1976}

Mieczyslaw Altman, Contractor directions, directional contractors and

directional contractions for solving equations . .................. 1

Michael Peter Anderson, Subgroups of finite index in profinite groups .........

Zvi Arad, Abelian and nilpotent subgroups of maximal order of groups of odd order

John David Baildon and Ruth Silverman, On starshaped sets and Helly-type theorems ..........................................

John W. Baker and R. C. Lacher, Some mappings which do not admit an

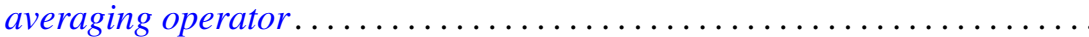

Joseph Barback, Composite numbers and prime regressive isols . . . . . . . . . .

David M. Boyd, Composition operators on $H^{p}(A) \ldots \ldots \ldots \ldots \ldots \ldots \ldots$

Maurice Chacron, Co-radical extension of PI rings . . . . . . . . . . . . .

Fred D. Crary, Some new engulfing theorems . . . . . . . . . . . . . . .

Victor Dannon and Dany Leviatan, A representation theorem for convolution transform with determining function in $L^{p} \ldots \ldots \ldots \ldots \ldots \ldots \ldots \ldots \ldots \ldots \ldots \ldots \ldots \ldots$

Mahlon M. Day, Lumpy subsets in left-amenable locally compact

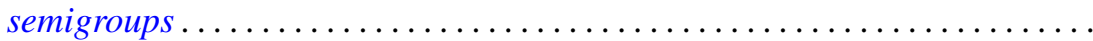

Michael A. Gauger, Some remarks on the center of the universal enveloping algebra of a classical simple Lie algebra . .

David K. Haley, Equational compactness and compact topologies in rings

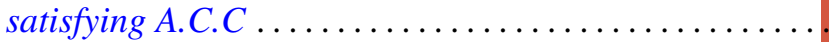

Raymond Heitmann, Generating ideals in Prüfer domains .

Gerald Norman Hile, Entire solutions of linear elliptic equations with

Laplacian principal part. .

Richard Oscar Hill, Moore-Postnikov towers for fibrations in which $\pi_{1}$ (fiber) is non-abelian

John Rast Hubbard, Approximation of compact homogeneous maps . .

Russell L. Merris, Relations among generalized matrix functions . .

V. S. Ramamurthi and Edgar Andrews Rutter, On cotorsion radicals ...

Ralph Tyrrell Rockafellar and Roger Jean-Baptiste Robert Wets, Stochastic

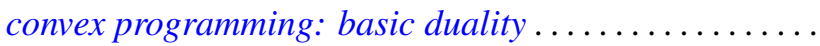

Alban J. Roques, Local evolution systems in general Banach spaces ..

I. Bert Russak, An indirect sufficiency proof for problems with bounded state variables.

Richard Alexander Sanerib, Jr., Ultrafilters and the basis property. .

H. A. Seid, The decomposition of multiplication operators on $L_{p}$-spaces . .

Franklin D. Tall, The density topology .................. 